Sports Science

\title{
Reference values, intrarater reliability, and measurement error for the closed kinetic chain upper extremity stability test and upper quarter y balance test in young adults
}

\author{
Anna Luiza Teixeira $^{1}$ (D), Anamaria Siriani de Oliveira $^{2}$ (D), Nathália Alves Rodrigues ${ }^{1}$ (D), \\ Guilherme Augusto Santos Bueno ${ }^{3}$ (D), Maria Eduarda Oliveira Novais ${ }^{1}$ (D), \\ Rayanne de Paula Moreira ${ }^{1}$ (D), Thiago Vilela $\operatorname{Lemos}^{4}$ (D), João Paulo Chieregato Matheus ${ }^{3}$ (D), \\ José Roberto de Souza Júnior ${ }^{3}$ \\ ${ }^{I}$ Faculdade Evangélica de Ceres, Departamento de Fisioterapia, Ceres, GO, Brazil; \\ ${ }^{2}$ Universidade de São Paulo, Faculdade de Medicina de Ribeirão Preto, Departamento de \\ Ciências da Saúde, Ribeirão Preto, SP, Brazil; ${ }^{3}$ Universidade de Brasília, Faculdade de \\ Ceilândia, Programa de Pós-Graduação em Ciências e Tecnologias em Saúde, Brasília, DF, \\ Brazil; ${ }^{4}$ Universidade Estadual de Goiás, Campus Metropolitano de Goiânia, Departamento de \\ Fisioterapia, Goiânia, GO, Brazil.
}

Associated Editor: Ricardo Barbieri (D), Estácio UniSEB, Ribeirão Preto, SP, Brazil. E-mail:

barbieri_ef@hotmail.com.

\begin{abstract}
Aims: To describe reference values for the Closed Kinetic Chain Upper Extremity Stability Test (CKCUEST) and Upper Quarter Y Balance Test (UQYBT) in young adults, and to determine whether there were differences in both tests based on gender and age. Intrarater reliability and measurement error were also assessed. Methods: Testretest design with a sample of 146 young adults. The CKCUEST (number of touches, normalized score, and power) and the UQYBT (normalized reach in the medial, superolateral, and inferolateral direction, and the composite score) were used. Mean and standard deviation were calculated for both tests. A linear and a mixed regression model were applied to determine significant differences in test scores. Reliability was determined using Intraclass Correlation Coefficient (ICC) and error measurement through Standard Error of Measurement (SEM) and Smallest Detectable Change (SDC). Results: Normative values were established and divided by gender and age. Men presented greater scores regarding the number of touches, power score, superolateral and inferolateral reaches. Good intrarater reliability was found for both tests. In the CKCUEST, the SEM and SDC values were 1 and 3 touches. In the UQYBT, the SEM values ranged from 3 to $7 \mathrm{~cm}$, while the SDC ranged from 8 to $19 \mathrm{~cm}$. Conclusion: Normative data were provided and men presented greater scores than women. Good intrarater reliability was found and values of SEM and SDC were established. Clinicians must use both tests in clinical practice since different aspects of the upper quarter are assessed.
\end{abstract}

Keywords: upper extremity, physical functional performance, outcome assessment.

\section{Introduction}

Physical performance tests are simple tools that do not require great expertise to apply and are recommended as a method of screening subjects who may develop musculoskeletal injuries. These tests are used mainly in the sports environment, as they do not require expensive equipment and can be applied in varied situations ${ }^{1,2}$. Among the physical performance tests for the upper limbs, the Closed Kinetic Chain Upper Extremity Stability Test (CKCUEST) and the Upper Quarter Y Balance Test (UQYBT) are the most studied ${ }^{3-6}$.

The CKCUEST is a closed kinetic chain test with fast execution, performed in the push-up position that evaluates functional performance bilaterally and is capable to predict shoulder injuries ${ }^{6-9}$. The test consists of counting how many times the subject performs alternating touches on the opposite hand over $15 \mathrm{~s}$ and requires concentric and eccentric muscle strength of the shoulder complex. The number of touches presented a moderate to strong correlation with peak torque of the shoulder external/internal rotators in previous studies ${ }^{10}$.

Meanwhile, the UQYBT is a closed kinetic chain test with slow execution, performed in the push-up position, which evaluates functional performance unilaterally ${ }^{3,5,11}$. The test consists in reach a maximum range in three directions (medial, superolateral, and inferolateral) named according to the stationary hand, and 
focuses on other components including balance, proprioception, and mobility of the thoracic spine and scapula ${ }^{5}$. The scores of this test present a small correlation with lateral trunk endurance ${ }^{7}$.

Despite the applicability of these tests, it is well documented that there is less evidence about functional performance tests of the upper limbs than the lower limbs ${ }^{2}$. The presence of reference values in different populations would provide information about the function of the upper quarter, the effectiveness of specific kinds of training, and the optimal time to return to activities/sport. In addition, information regarding measurement properties in different populations would help clinicians to use reliable tools, and to identify results related to measurement error or significant improvement ${ }^{6}$.

Therefore, the primary purpose of the study was to describe reference values for the Closed Kinetic Chain Upper Extremity Stability Test and Upper Quarter Y Balance Test in a sample of Brazilian adults and to determine whether there were differences in both tests based on gender and age. The secondary purpose of the study was to assess intrarater reliability and measurement error. Our main hypothesis is that men and younger adults present greater scores in both tests. Also, good to excellent intrarater reliability and the absence of systematic error would be found.

\section{Methods}

\section{Type of study}

A test-retest design was carried out according to the recommendations of STROBE (Strengthening the Reporting of Observational Studies in Epidemiology) and COSMIN (COnsensus-based Standards for the selection of health Measurement Instruments). The study was approved by the Ethics Committee on Research with Humans of the UniEvangélica (CAAE: 03915818.2.0000.5076). Informed consent was obtained in accordance with the Helsinki Declaration and local resolution.

\section{Participants}

A convenience sample of 146 young adults participated in the study. They were divided according to gender $(63=$ male versus $83=$ female $)$ and age category $(126=18-25$ versus $20=26-40$ years $)$. The inclusion criteria were (I) age between 18 and 40 years. (II) both sexes. The exclusion criteria were (I) presence of previous injuries in the upper limbs; (II) history of previous surgeries in the upper limbs; (III) presence of cardiovascular, orthopedic, neurological, or rheumatological diseases that could compromise the performance in the functional tests.

\section{Outcomes}

The average number of touches, number of touches normalized by height, power score of the CKCUEST, reaches in the medial, inferolateral, and superolateral directions normalized by the upper limb length, and the composite score of the UQYBT according to gender and age were the primary outcomes of the study. The intrarater reliability and measurement error for the CKCUEST and UQYBT scores were the secondary outcomes of the study. The reference values were provided considering the total sample $(\mathrm{n}=146)$, while the intrarater reliability was calculated considering part of the sample $(n=35)$.

\section{Study protocol}

Data collection began with the application of a questionnaire to obtain identification data and general information such as age, weight, height, body mass index, and length of the upper limbs. The length of the upper limbs was assessed with the participant standing, ninety-degree shoulder abduction, elbow extension, and wrist/hand in a neutral position. The measurement was made from the spinous process of $\mathrm{C} 7$ to the distal phalanx of the third finger ${ }^{3}$.

After this stage, the tests were performed. Before the tests were executed, the participants received information about how they were to be performed. The participants performed these tests with two researchers (A.L.T and N.A.R), both of whom were previously trained to apply the tests. After seven days, both tests were performed in the same conditions to assess intrarater reliability.

For CKCUEST, the participants were positioned in the push-up position with lower limbs extended and with the arms out perpendicular at a distance of $92 \mathrm{~cm}$. Participants performed alternate taps on the opposite hand as quickly as possible for $15 \mathrm{~s}$. The number of touches for $15 \mathrm{~s}$ was assessed. Four attempts were allowed with an interval of $30 \mathrm{~s}$ between them, the first being for practice, and the average of the last three for analysis. The men performed the test in the push-up position with the lower limbs extended, while the women performed in the same position with the lower limbs flexed. If the participant did not reach the predetermined mark or left the position, the repetition was not validated ${ }^{6}$. The normalized score was obtained by dividing the number of touches by subject height (in), and the power score was obtained by multiplying the average number of touches by $68 \%$ of the subject's body weight in $\mathrm{kg}$ (a percentage that corresponds to the weight of the arms, head, and trunk) divided by 15 (elapsed test time in $\mathrm{s})^{6,12}$.

For UQYBT, the participants were positioned in the push-up position with the upper limbs extended. During UQYBT, one hand remained fixed while the other reached a maximum range in three directions (medial, superolateral, and inferolateral) named according to the sta- 
tionary hand $(\mathrm{cm})$. Four attempts were allowed with an interval of $30 \mathrm{~s}$ between them, the first being for practice, and the average of the last three for analysis. The UQYBT was done in an adapted way using measuring tapes ${ }^{13}$. Men and women performed the test in the same way, and they were instructed to remain with their feet supported in the initial position throughout the test. The repetition was considered invalid when either the stationary hand or feet changed their position during the test or if the reaching hand performed weight unloading and not a light touch on the tape. A normalized score was obtained dividing the reach in each direction by the upper limb length $(\mathrm{cm})$ multiplied by $100^{5}$. The composite score was calculated by taking the total excursion distance (medial + superolateral + inferolateral) and dividing it by 3 times the upper limb length $(\mathrm{cm})^{7}$.

\section{Statistical analysis}

Data were analyzed using SPSS software (version 23.0; SPSS, Chicago, IL, USA). Data normality and homogeneity of variance were tested using the ShapiroWilk and Levene's tests, respectively. Mean and standard deviation were calculated for the anthropometric data, and the CKCUEST and UQYBT scores. For the anthropometric data and CKCUEST, a linear regression model was applied considering "gender" (male or female), and "age category" (18-25 or 26-40) as fixed factors in order to determine significant differences between these aspects. Additionally, side differences (right or left) were also checked for the UQYBT test using a linear mixed model by adding a fixed factor "side". The anthropometric data that were different across the subgroups were included in the model as covariates. For all variables, only the highest significant interaction-effect (or mean effect in absence of an interaction effect) was used in the model for interpreting the results ${ }^{3}$. Sidak's post hoc was used to make pairwise comparisons.

The intrarater reliability of the tests was assessed using the Intraclass Correlation Coefficient (ICC) with a mixed two-way model, evaluation of the same examiner at different times, and absolute agreement. ICC values greater than 0.70 were considered as the minimum standard for good reliability ${ }^{14}$. Measurement Error was calculated using the Standard Error of Measurement (SEM) and the Smallest Detectable Change (SDC). The Standard Error of Measurement (SEM) was calculated using the following formula: $\mathrm{SEM}=\mathrm{SD} \times \sqrt{ }$ 1-ICC where SEM $=$ Standard Error of Measurement; SD $=$ Standard deviation of the variable; ICC $=$ Intraclass Correlation Coefficient. The Smallest Detectable Change (SDC) was calculated as follows: SDC $=$ SEM $\times 1.96 \times \sqrt{ } 2$ where SDC $=$ Smallest Detectable Change; SEM $=$ Standard Error of Measurement. Bland-Altman Plots (BAPs) were used to verify the absolute agreement between assessments from the scatter plot between the difference of the two assessments and the average of the two evaluations. Bias and 95\% limits of agreement were used to determine the accuracy of these measures. Also, a linear regression analysis was conducted to test the null hypothesis. In the presence of missing items, information collected was considered in the analysis. A significance level of $p<0.05$ was used.

\section{Results}

The participants had a mean age of 21.83 (3.58) years, a body mass of $66.69(14.71) \mathrm{kg}$, a height of 168.41 $(8.68) \mathrm{cm}$, and a Body Mass Index of $23.32(4.09) \mathrm{kg} / \mathrm{m}^{2}$. An effect of the interaction between gender and age category was found only for height $[F=4.09 ; p=0.045]$. Post hoc test showed that males have a higher height than females in the age categories $(\mathrm{p}<0.001)$, and that females with 26-40 years have higher height compared to 1825 years $(p=0.030)$. Also, an effect of the gender was found for body mass $[\mathrm{F}=41.39 ; \mathrm{p}<0.001]$, and body mass index $[\mathrm{F}=8.42 ; \mathrm{p}=0.004]$, with men presenting greater values. No significant effect was found for age (Table 1).

\section{References values for the CKCUEST according to gender and age}

Due to the lack of influence of the anthropometric data on the scores of the CKCUEST $(p>0.05)$, these variables were not considered in the final statistical models. An effect of the interaction between gender and age category was found for the power score $[\mathrm{F}=5.76$; $\mathrm{p}=0.018]$. Post hoc test showed that males have higher scores than females in the age categories $(\mathrm{p}<0.001)$, and that males with 26-40 years have higher scores compared

Table 1 - Mean and standard deviation of the anthropometric characteristics according to gender and age category $(\mathrm{n}=146)$.

\begin{tabular}{|c|c|c|c|}
\hline & Total $(n=146)$ & Women $(n=83)$ & $\operatorname{Men}(n=63)$ \\
\hline \multicolumn{4}{|l|}{ Age (years) } \\
\hline $18-25$ years & $20.64(1.69)$ & $20.75(1.68)$ & $20.48(1.70)$ \\
\hline $26-40$ years & $29.35(3.23)$ & $28.66(3.16)$ & $29.90(3.33)$ \\
\hline \multicolumn{4}{|c|}{ Body Mass (kg) } \\
\hline $18-25$ years & $66.15(14.50)$ & $60.28(11.53)$ & 74.51 (14.28) \\
\hline $26-40$ years & 70.05 (15.94) & $56.55(7.63)$ & 81.09 (11.75) \\
\hline \multicolumn{4}{|l|}{ Height (cm) } \\
\hline $18-25$ years & $168.38(8.53)$ & $163.62(6.49)$ & $175.17(6.19)$ \\
\hline $26-40$ years & $168.55(9.80)$ & $158.88(3.40)$ & $176.45(4.52)$ \\
\hline \multicolumn{4}{|l|}{ BMI $\left(\mathrm{kg} / \mathrm{m}^{2}\right)$} \\
\hline $18-25$ years & $23.15(4.11)$ & $22.40(3.72)$ & $24.23(4.42)$ \\
\hline $26-40$ years & $24.40(3.95)$ & $22.33(3.04)$ & $26.09(3.91)$ \\
\hline
\end{tabular}

BMI = Body Mass Index. 
to $18-25$ years $(\mathrm{p}=0.028)$. Also, an effect of the gender was found for the number of touches $[\mathrm{F}=4.32$; $\mathrm{p}=0.039)$, with men presenting greater scores than females. No significant effect was found for the normalized score $(\mathrm{p}>0.05)$ (Table 2).

References values for the UQYBT according to gender and age

Due to the lack of influence of the anthropometric data on the scores of the UQYBT $(p>0.05)$, these variables were not considered in the final statistical models. An effect of the gender was found for the superolateral $[\mathrm{F}=37.23 ; \mathrm{p}<0.001]$, and inferolateral reach $[\mathrm{F}=11.07$; $\mathrm{p}=0.001]$, with men presenting greater scores. No significant effect was found for the medial reach, and composite score $(\mathrm{p}>0.05)$ (Table 3$)$.

\section{Intrarater reliability and measurement error for the CKCUEST and UQYBT}

Good intrarater reliability was found for the CKCUEST $(I C C=0.82)$ and UQYBT (Right: Medial - ICC = 0.83; Superolateral - ICC $=0.84$; Inferolateral - ICC $=0.77$; Left: Medial - ICC $=0.79$; Superolateral - ICC $=0.89$; Inferolateral - ICC $=0.74)$. In the CKCUEST, the SEM and SDC values were 1 and 3 touches. In the UQYBT, the SEM values ranged from 3 to $7 \mathrm{~cm}$, while the SDC ranged from 8 to $19 \mathrm{~cm}$ (Table 4). According to the Bland-Altman Plots and the regression analysis performed, it is possible to observe the absence of systematic error between assessments $(\mathrm{p}>0.05)$ for the CKCUEST (Figure 1) and UQYBT (Figures 2-3).

\section{Discussion}

Normative data in a sample of Brazilian adults was provided according to gender and age, and it was possible

Table 2 - Mean and standard deviation for the Closed Kinetic Chain Upper Extremity Stability Test scores according to gender and age category $(n=146)$.

\begin{tabular}{lccc}
\hline & Total $(\mathbf{n}=\mathbf{1 4 6})$ & Women $(\mathbf{n}=\mathbf{8 3})$ & Men $(\mathbf{n}=\mathbf{6 3})$ \\
\hline Number of Touches & & & \\
18-25 years & $18.80(3.45)$ & $18.68(3.51)$ & $18.98(3.39)$ \\
26-40 years & $19.05(3.13)$ & $17.33(3.35)$ & $20.45(2.20)$ \\
Normalized score & & & \\
$18-25$ years & $0.28(0.05)$ & $0.29(0.05)$ & $0.27(0.05)$ \\
$26-40$ years & $0.28(0.04)$ & $0.27(0.05)$ & $0.29(0.03)$ \\
Power & & & \\
$18-25$ years & $56.48(16.55)$ & $51.07(13.87)$ & $64.19(17.10)$ \\
$26-40$ years & $61.45(19.73)$ & $44.67(11.92)$ & $75.19(12.83)$ \\
\hline
\end{tabular}

Normalized score $=$ number of touches/height in in; Power $=($ number of touches $\mathrm{x} 68 \%$ body weight in $\mathrm{kg}) / 15$.
Table 3 - Mean and standard deviation for the Upper Quarter Y Balance Test scores according to gender and age category $(n=146)$.

\begin{tabular}{lcccccc}
\hline & \multicolumn{2}{c}{ Total $(\mathbf{n}=\mathbf{1 4 6})$} & \multicolumn{2}{c}{ Women $(\mathbf{n = 8 3})$} & \multicolumn{2}{c}{ Men $(\mathbf{n}=\mathbf{6 3})$} \\
\hline Medial & Right & Left & Right & Left & Right & Left \\
18-25 years & 101.80 & 103.82 & 101.86 & 101.89 & 101.72 & 106.69 \\
& $(13.52)$ & $(14.44)$ & $(13.67)$ & $(14.88)$ & $(13.46)$ & $(13.42)$ \\
& 102.34 & 105.83 & 102.56 & 111.43 & 102.13 & 101.25 \\
& $(16.69)$ & $(16.85)$ & $(18.52)$ & $(14.28)$ & $(15.76)$ & $(18.02)$
\end{tabular}

Superolateral

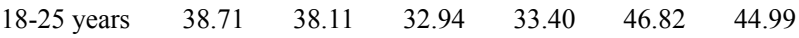

$\begin{array}{llllll}(13.47) & (14.52) & (10.82) & (13.54) & (12.72) & (13.22)\end{array}$

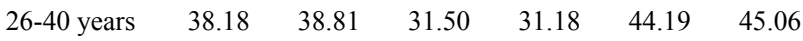

$\begin{array}{llllll}(10.03) & (13.46) \quad(8.26) \quad(7.45) \quad(7.46) \quad(14.30)\end{array}$

Inferolateral

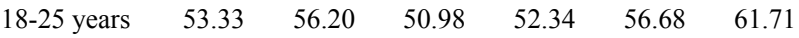

$\begin{array}{llllll}(15.64) & (16.66) & (16.41) & (16.51) & (13.98) & (15.44)\end{array}$

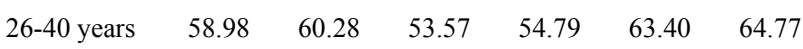

$\begin{array}{llllll}(12.35) & (11.09) & (8.30) & (8.10) & (13.67) & (11.48)\end{array}$

Composite

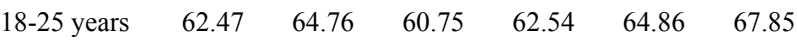

$\begin{array}{llllll}(12.85) & (13.16) & (13.37) & (12.17) & (11.81) & (13.96)\end{array}$

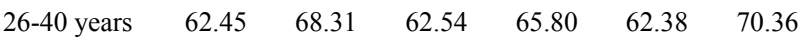

$\begin{array}{llllll}(14.54) & (10.30) \quad(8.91) \quad(7.38) \quad(18.39) \quad(12.16)\end{array}$

Medial, Superolateral, and Inferolateral scores $=($ reach in $\mathrm{cm} /$ upper limb length in $\mathrm{cm}) \times 100$; Composite score $=($ total excursion in $\mathrm{cm} / 3 \times$ upper limb length in $\mathrm{cm}) \times 100$.

to note that male participants presented greater scores regarding the number of touches, power score, superolateral and inferolateral reaches. Good intrarater reliability was found for both tests. In the CKCUEST, the SEM and

Table 4 - Intrarater reliability: Intraclass Correlation Coefficient with 95\% Confidence Intervals, Standard Error of Measurement, and Smallest Detectable Change for the Closed Kinetic Chain Upper Extremity Stability Test, and the Upper Quarter Y Balance Test scores $(n=35)$.

\begin{tabular}{lcccc}
\hline & & ICC (95\%) & SEM & SDC \\
\hline Number of touches & & $0.82(0.55-0.92)$ & 1 & 3 \\
Medial & Right & $0.83(0.67-0.92)$ & 5 & 13 \\
& Left & $0.79(0.58-0.89)$ & 4 & 11 \\
Superolateral & Right & $0.84(0.70-0.92)$ & 3 & 8 \\
& Left & $0.89(0.78-0.95)$ & 3 & 8 \\
Inferolateral & Right & $0.77(0.43-0.90)$ & 7 & 19 \\
& Left & $0.74(0.50-0.87)$ & 7 & 19 \\
\hline
\end{tabular}

IC 95\% = Confidence Interval of 95\%; SEM = Standard Error of Measurement; $\mathrm{SDC}=$ Smallest Detectable Change; ICC $=$ Intraclass Correlation Coefficient - poor reliability $(<0.50)$, moderate reliability $(0.50$ $0.75)$, good reliability $(0.75-0.90)$, excellent reliability $(>0.90)$. 


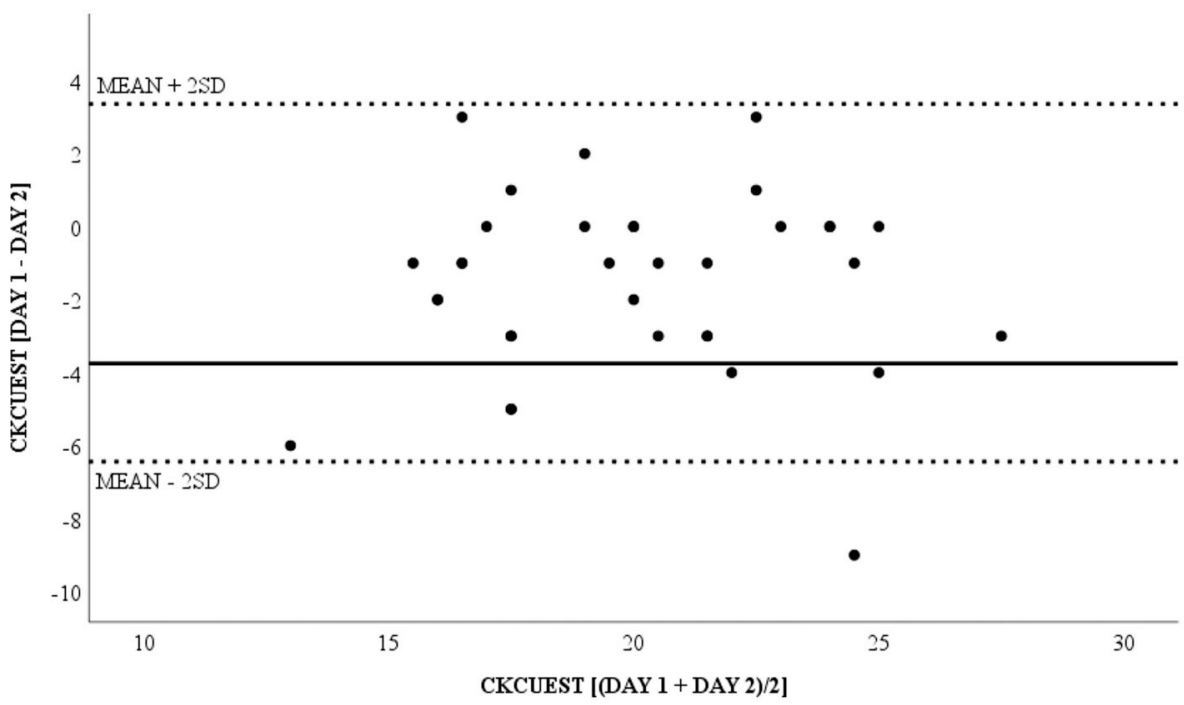

Figure 1 - Bland-Altman Plot for the number of touches in the Closed Kinetic Chain Upper Extremity Stability Test. The straight line represents bias and dotted lines $95 \%$ limits of agreement.

SDC values were 1 and 3 touches. In the UQYBT, the SEM values ranged from 3 to $7 \mathrm{~cm}$, while the SDC ranged from 8 to $19 \mathrm{~cm}$. The absence of systematic error was found for both tests.

The reference values for the number of touches in healthy volunteers were similar when compared with two studies $^{7,15}$ and lower when compared with other two ${ }^{6,12}$. Regarding the normalized score, it was similar with one study $^{15}$ and lower when compared to another ${ }^{6}$. The power score was lower when compared to the previous studies $^{6,15}$. As expected, CKCUEST values were lower when compared to studies that assessed athletes ${ }^{3,16}$. The effect of gender found in the number of touches was similar when compared with studies that assessed athletes ${ }^{3,16}$, however, disagree with studies conducted with healthy volunteers $^{6,7}$. Similar to our study, no effect of age was found in overhead athletes $(18-25 \times 26-33)^{3}$. The study of Tucci et al. ${ }^{6}$ did not find any effect of gender in the normalized or power score, while we found an effect of the interaction between gender and age category in the power score.

The reference values for the medial reach were similar with studies that assessed healthy volunteers ${ }^{5}$ and throwing athletes ${ }^{4}$. Lower values were found compared to athletes from different sports ${ }^{16}$. The values of the superolateral and inferolateral reaches, and the composite score were lower when compared to healthy volunteers ${ }^{5}$ or athletes $^{4,16}$. In addition, our results were lower than two studies that assessed healthy volunteers and overhead athletes, however, these studies did not present the normalized score ${ }^{3,7}$. An effect of the gender in the medial and composite scores was not found in our study, similar results were found in healthy volunteers ${ }^{5}$, however, this effect was reported in athletes ${ }^{3,16}$. Differences in the superolateral reach were presented in overhead athletes of older age categories, but not in throwing athletes ${ }^{16}$ or healthy volunteers ${ }^{5}$. Differences in the inferolateral reach were reported only in athletes ${ }^{3,16}$. Age differences were found only for the superolateral reach in female volleyball athletes $^{3}$. Also, differences between sides were reported only for the inferolateral and composite scores (non-dominant $>$ dominant $)^{3}$.

As presented above, there is not a consensus about normative values and differences regarding gender, age, or dominance (UQYBT only). In this context, it is important to note that the discussions about these topics are based only on unique values and not on a range of values ${ }^{6}$. Differences regarding population, familiarization, types of equipment, execution of the tests, number of trials, learning effect, motivation, and variables selected for analysis may explain the differences found in the literature.

Good intrarater reliability was found for the number of touches in the CKCUEST and all directions in the UQYBT. These results corroborate with previous studies that assessed healthy volunteers ${ }^{5-7,12,17,18}$ and athletes from different sports ${ }^{3,4,16}$. In the UQYBT, the superolateral reach presented the best ICC values; similar results were found in both healthy volunteers ${ }^{5}$ and athletes $^{3}$. In relation to measurement error, for the number of touches in the CKCUEST the SEM was 1 touch, and the SDC was 3 touches, similar results were found in adolescents $(\mathrm{SEM}=2.17 ; \mathrm{SDC}=6.01)^{18}$ and young adults $(\mathrm{SEM}=0.93-2.76 \text {; } \mathrm{SDC}=2.05-4.25)^{6,17}$. For the UQYBT, SEM values varied between 3 and $7 \mathrm{~cm}$, and SDC between 8 and $19 \mathrm{~cm}$, results that are higher than the SEM and SDC reported in healthy volunteers ${ }^{5}(\mathrm{SEM}=2.2-2.9$; $\mathrm{SDC}=6.1-8.1)$ and throwing athletes ${ }^{4}(\mathrm{SEM}=1.41-1.77$; $\mathrm{SDC}=3.91-4.91)$, but still without systematic error. 

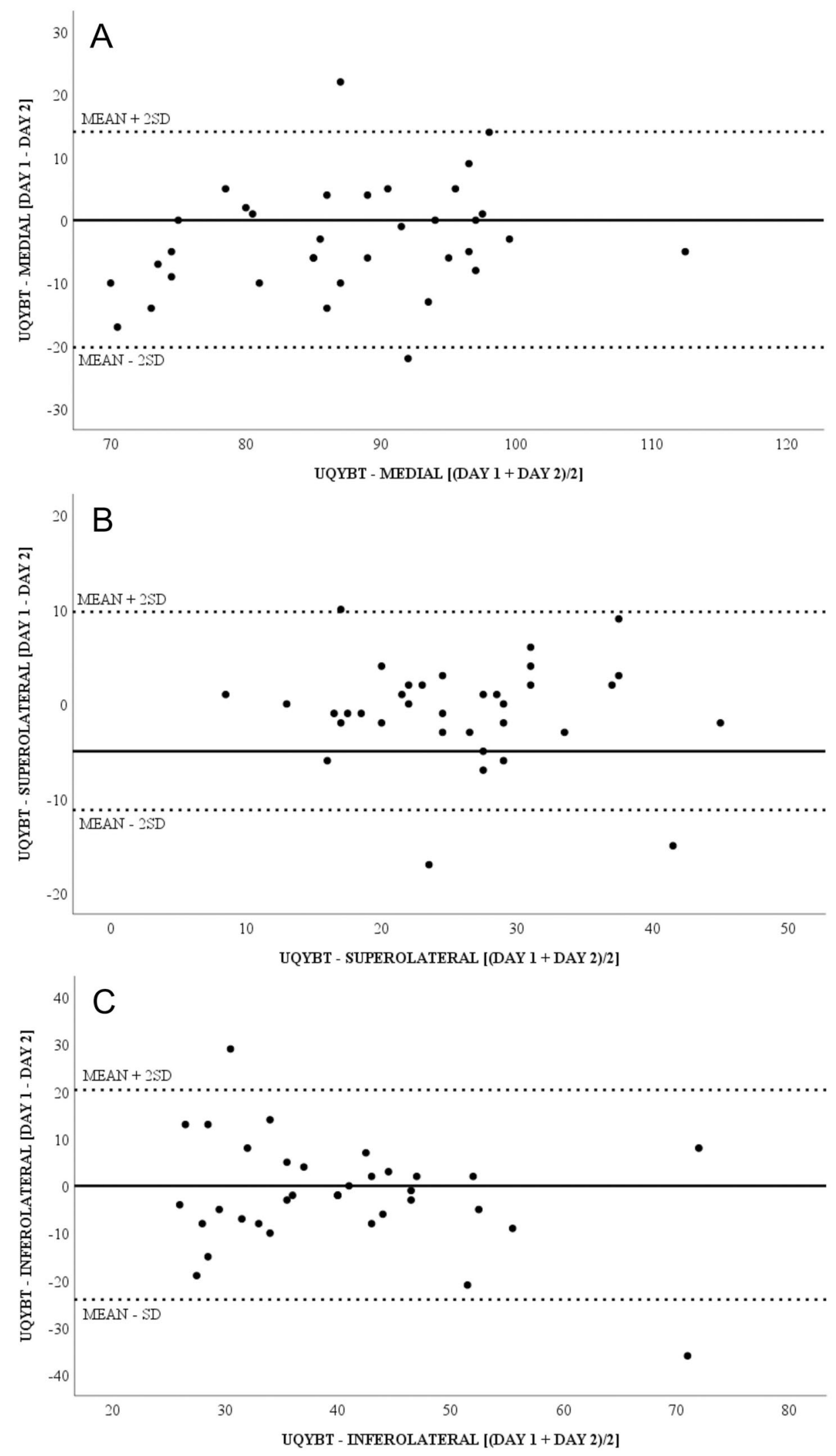

Figure 2 - Bland-Altman Plot for the three directions of the Upper Quarter Y Balance Test in the right upper limb: A) medial; B) superolateral; C) inferolateral. The straight line represents bias and dotted lines $95 \%$ limits of agreement. 

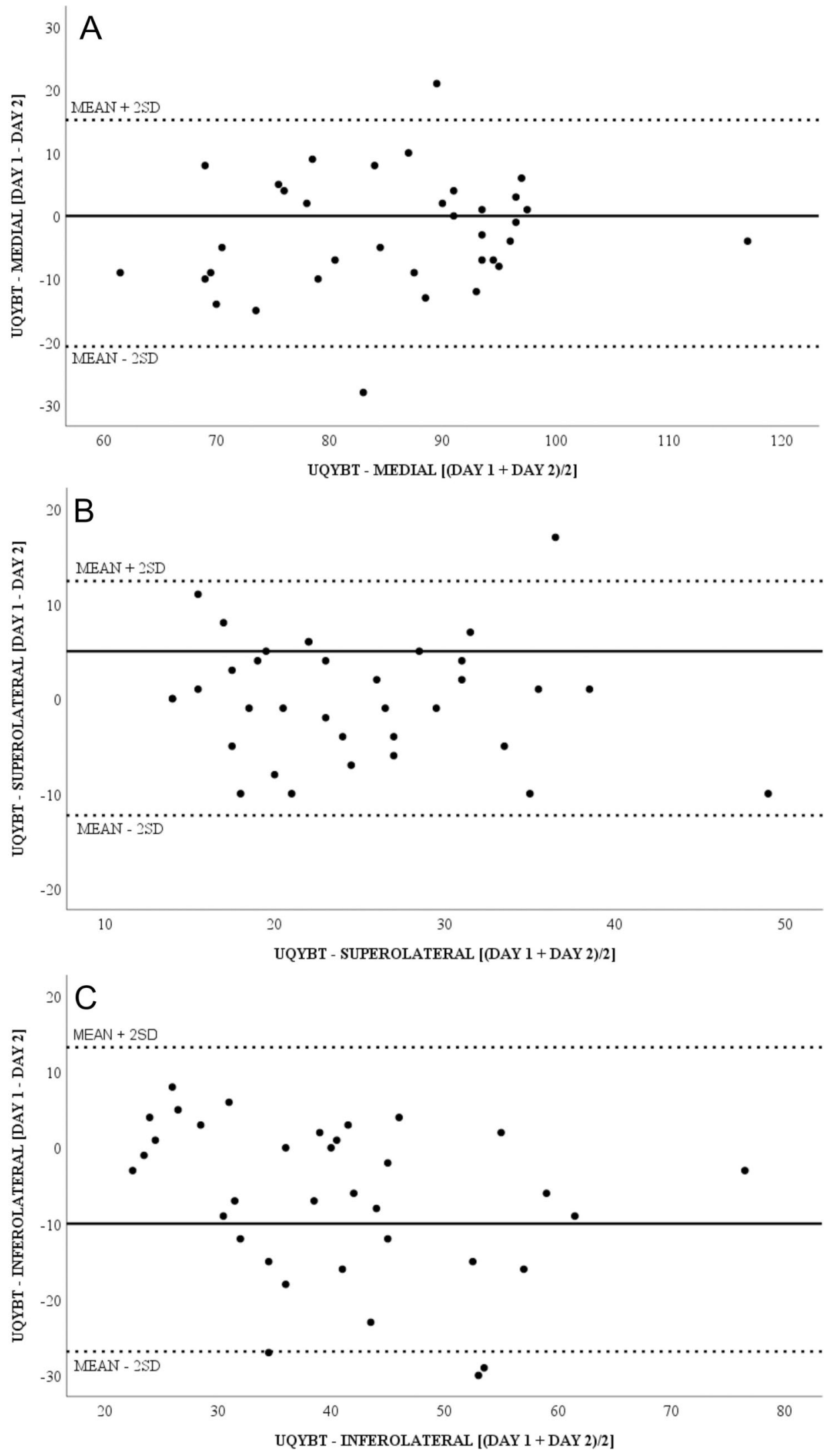

Figure 3 - Bland-Altman Plot for the three directions of the Upper Quarter Y Balance Test in the left upper limb: A) medial; B) superolateral; C) inferolateral. The straight line represents bias and dotted lines $95 \%$ limits of agreement. 
Applicability of upper quarter physical performance tests in young adults

The CKCUEST and the UQYBT are easy, low-cost, quick to apply, and reliable tools that can be used to assess the functionality of the upper limbs. Therefore, the innovation of this study consists of in to provide information regarding the normative data according to gender and age in a sample of Brazilian healthy volunteers. In addition, SEM and SDC values for both tests were provided. The SEM values are important to differentiate values that may be related solely to measurement error ${ }^{18}$. With the data of the smallest detectable change, practitioners will be able to compare the results within training and verify any clinically significant improvement ${ }^{6}$. It is suggested that both tests should be applied together in clinical practice since they assess different constructs of the upper quarter.

The limitations of the study must be highlighted. Multiple tests must be conducted to promote an adequate performance by the participant, and in our study, only a single familiarization session was used. It was not possible to stratify participants according to anthropometric characteristics. This aspect can influence the results of the tests, especially because the equations used to calculate the Normalized Score and Power depend on height and body weight respectively ${ }^{6}$. The tests were performed only in very sedentary participants and stratification on fitness level was not conducted. Finally, it was not possible to provide reliability and measurement error values according to gender and age since these analyses were conducted in part of the sample.

\section{Conclusions}

In conclusion, reference values for the CKCUEST and UQYBT were provided according to gender and age in a sample of Brazilian adults. In general, men presented greater scores than women in the number of touches, power score, superolateral and inferolateral reaches, and composite scores. Good intrarater reliability was found and values of SEM and SDC were established. The absence of systematic error was found. Clinicians must use both tests in clinical practice since different aspects of the upper quarter are assessed. Future studies that evaluate the applicability of these instruments in relation to different populations, the capability of these tests to predict upper extremity injuries, and the power to determine return to normal activities or sports must be carried out. Also, the relationship with aspects of the kinetic chain must be investigated.

\section{References}

1. Hegedus EJ, McDonough S, Bleakley C, Cook CE, Baxter GD. Clinician-friendly lower extremity physical performance measures in athletes: a systematic review of measurement properties and correlation with injury, part 1 . The tests for knee function including the hop tests. Br J Sports Med. 2014;49(10):642-8. DOI.
2. Tarara DT, Fogaca LK, Taylor JB, Hegedus EJ. Clinicianfriendly physical performance tests in athletes, part 3: a systematic review of measurement properties and correlations to injury for tests in the upper extremity. Br J Sports Med. 2015;50(9):545-51. DOI.

3. Borms D, Cools A. Upper-extremity functional performance tests: reference values for overhead athletes. Int J Sports Med. 2018;39(06):433-41. DOI.

4. Borms D, Maenhout A, Cools AM. Upper quadrant field tests and isokinetic upper limb strength in overhead athletes. J Athl Train. 2016;51(10):789-96. DOI.

5. Gorman PP, Butler RJ, Plisky PJ, Kiesel KB. Upper quarter Y balance test. J strength Cond Res. 2012;26(11):3043-8. DOI.

6. Tucci HT, Martins J, Sposito GC, Camarini PMF, de Oliveira AS. Closed Kinetic Chain Upper Extremity Stability test (CKCUES test): a reliability study in persons with and without shoulder impingement syndrome. BMC Musculoskelet Disord. 2014;15(1). DOI.

7. Westrick RB, Miller JM, Carow SD, Geber JP. Exploration of the $y$-balance test for assessment of upper quarter closed kinetic chain performance. Int J Sports Phys Ther. 2012;7 (2):139-47.

8. Callaway A, Peck J, Ellis S, Williams J. A randomised observational study of individualised variations in the start position of the closed-kinetic chain upper extremity stability test. Phys Ther Sport. 2020;41:16-22. DOI

9. Pontillo M, Spinelli BA, Sennett BJ. Prediction of in-season shoulder injury from preseason testing in Division I collegiate football players. Sports Health. 2014;6(6):497-503. DOI.

10. Lee D-R, Kim LJ. Reliability and validity of the closed kinetic chain upper extremity stability test. J Phys Ther Sci. 2015;27(4):1071-3. DOI

11. Butler RJ, Heather SM, Douglass B, Kyle BK, Phillip JP, Moorman CT, et al. Bilateral differences in the upper quarter function of high school aged baseball and softball players. Int J Sports Phys Ther. 2014;9(4):518-24.

12. Goldbeck TG, Davies GJ. Test-retest reliability of the closed kinetic chain upper extremity stability test: a clinical field test. J Sport Rehabil. 2000;9(1):35-45. DOI

13. Cramer J, Quintero M, Rhinehart A, Rutherford C, Nasypany A, May J, et al. Exploration of score agreement on a modified upper quarter y-balance test kit as compared to the upper quarter y-balance test. Int J Sports Phys Ther. 2017;12 (1):117-24.

14. Mokkink LB, Prinsen CA, Bouter LM, Vet HC, Terwee CB. The COnsensus-based Standards for the selection of health Measurement Instruments (COSMIN) and how to select an outcome measurement instrument. Braz J Phys Ther. 2016;20(2):105-13. DOI

15. Ellenbecker TS, Manske R, Davies GJ. Closed kinetic chain testing techniques of the upper extremities. Orthop Phys Ther Clin North Am. 2000;9:219-30.

16. Taylor JB, Wright AA, Smoliga JM, DePew JT, Hegedus EJ. Upper-extremity physical-performance tests in college athletes. J Sport Rehabil. 2016;25(2):146-54. DOI

17. Silva YA, Novaes WA, Dos Passos MHP, Nascimento VYS, Cavalcante BR, Pitangui ACR, et al. Reliability of the 
closed kinetic chain upper extremity stability test in young adults. Phys Ther Sport. 2019;38:17-22. DOI

18. Oliveira VMA, Pintangui ACR, Nascimento VYS, Silva HA, Passos MHP, Araújo RC. Test-retest reliability of the closed kinetic chain upper extremity stability test (CKCUEST) in adolescents: reliability of CKCUEST in adolescents. Int J Sports Phys Ther. 2017;12(1):125-32.

\section{Corresponding author}

José Roberto de Souza Júnior. Universidade de Brasília, Faculdade de Ceilândia, Programa de Pós-Graduação em
Ciências e Tecnologias em Saúde, Brasília, DF, Brazil. E-mail: joserobertofisio@gmail.com.

Manuscript received on June 10, 2021

Manuscript accepted on October 27, 2021

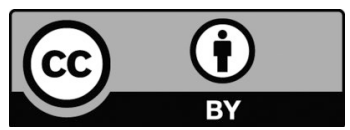

Motriz. The Journal of Physical Education. UNESP. Rio Claro, SP, Brazil - eISSN: 1980-6574 - under a license Creative Commons - Version 4.0 